\title{
Pills of PTEN? In and out for tumor suppression
}

\author{
Cell Research (2013) 23:1155-1156. doi:10.1038/cr.2013.103; published online 6 August 2013
}

\begin{abstract}
The tumor-suppressive activity of PTEN has always been attributed to its endogenous intracellular function. Recently two different groups have demonstrated that PTEN is secreted/ exported into the extracellular environment for uptake by recipient cells, and functions as a tumor suppressor in a cell non-autonomous manner.
\end{abstract}

The tumor suppressor PTEN is a key mediator of several intracellular signals. Together with its central role in regulating the activation of the protooncogenic PI3K/Akt signaling pathway in the cytoplasm as a lipid phosphatase, PTEN also functions as an essential component of the nuclear compartment, where it restrains cell cycle progression and favors chromosome stability [1-3].

Over the past 15 years, a number of in vivo and in vitro studies have highlighted the importance of PTEN functions within the cell, ranging from inhibition of cell growth and proliferation to promotion of apoptosis and tumor-suppressive metabolic states [4-6]. Accordingly, loss of PTEN has been found to provoke a dramatic imbalance in the cell, which is associated with developmental defects and tumorigenesis [4].

Remarkably, even as our knowledge of this tumor suppressor seemed exhaustive and its pathways were drawn in their entirety, two laboratories provided surprising new insights into the mode of action of this protein. In a recent paper in Science, Hopkins et al. [7] reported two major discoveries about an unexpected mechanism of action by the PTEN tumor suppressor.

Initially, the authors found that the canonical translation start site on PTEN
mRNA, ATG at bp 1032 , is preceded by an alternative initiation code, CTG at bp 513, whose expression predicts a longer PTEN variant, termed PTENLong. PTEN-Long is a 576 amino acid long enzyme with active phosphatase function toward the lipid target Phosphatidylinositol $(3,4,5)$-triphosphate, PIP3. PTEN-Long is endogenously expressed in human cell lines (reportedly HEK293 and T47D) and normal tissues (breast and brain), and reductions of its expression level have been observed in both human breast tumor samples and a mouse model of glioblastoma. Importantly, the authors revealed that the additional 173 amino acids uniquely found in PTEN-Long encode a secretion signal sequence that allows this enzyme to be secreted into the extracellular environment. Once secreted, PTEN-Long can be absorbed by recipient cells, where it functions as normal PTEN by inhibiting the PI3K pathway and inducing apoptosis. Thus, PTEN-Long acts as tumor suppressor in a cell non-autonomous manner.

In line with these findings, a previous report by Putz et al. [8] had already demonstrated that a secreted canonical PTEN — with an ATG at bp 1032 can be part of the intercellular signaling machinery, as the protein was found in exosomes, which are nanovesicles originating from endosomes and released into the extracellular space [9]. Indeed, endogenous and overexpressed PTEN was found to be actively exported outside the cytoplasmic membrane through exosomal trafficking, and later internalized by recipient cells with consequent functional effects (Figure 1, top panel).

Taken together, these results lead us to reconsider the conventional wisdom about PTEN, also raising a number of new and exciting questions. For instance, the identification of an alternative translation start site on PTEN mRNA points to an unexpectedly complex regulatory post-transcriptional network governing the expression of this major tumor suppressor, which from now on must be regarded as exhibiting both cell autonomous and non-autonomous properties. Yet the molecular mechanisms that favor the expression of conventional PTEN vs PTEN-Long remain to be determined, and no doubt promise important therapeutic implications. To this end, it is important to (1) perform a thorough molecular and biological characterization of PTEN-Long, aiming at a comprehensive analysis of its expression profile across tissues; (2) identify the mechanisms regulating the intercellular recognition of the enzyme between donor and recipient cells; (3) evaluate whether PTEN-Long affects cells in either close proximity (via a paracrine signal) or across long distances (in an endocrine scenario); and (4) understand whether PTEN-Long indeed displays a superior trafficking ability to a canonical PTEN (short isoform).

The generation and characterization of conditional Pten Knock-Out or Pten Knock-In mouse mutants will become extremely informative to assess the tissue-specific rate and the consequences of intercellar Pten trafficking.

However, there is more than one side to the story of the complicated relationship between PTEN and PTEN-Long. While wild-type PTEN and PTEN-Long both exert intra- and extra-cellular functions in suppressing tumorigenesis, the 

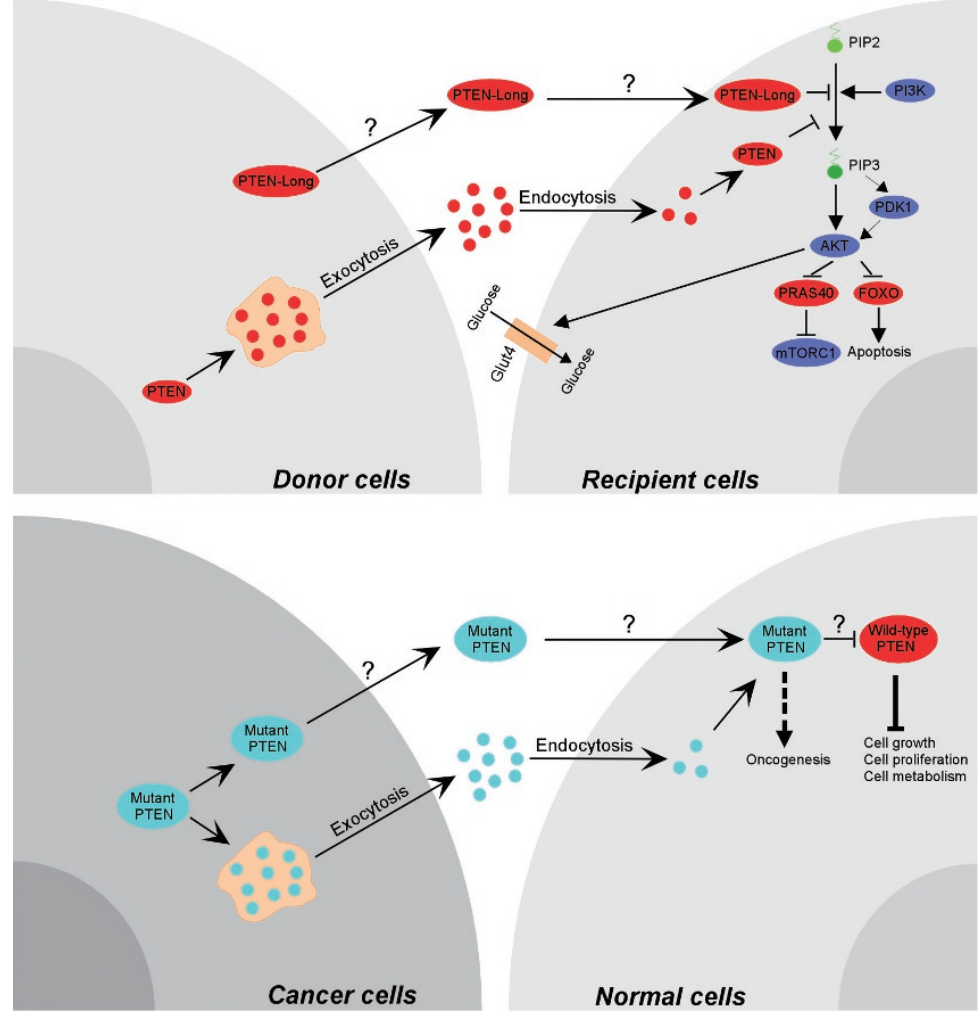

Figure 1 Cell non-autonomous effect of PTEN on tumor suppression. Conventional PTEN via exosomes, or PTEN-Long via an unknown mechanism, can be secreted from donor cells and enter recipient cells to antagonize PI3K signaling, induce apoptosis, and regulate cellular metabolism (Top panel). Likewise, exported mutant PTEN or secreted mutant PTEN-Long from cancer cells can enter the adjacent normal cells to interfere with wild-type PTEN functions, thus disrupting normal cell homeostasis and inducing a more pronounced tumor-prone condition (Bottom panel).

high incidence of germline and somatic PTEN mutations - either expressed as secreted PTEN-Long or -Short - can lead to unpredicted tumorigenic effects in a cell non-autonomous manner. Indeed, as sporadic PTEN mutations are commonly found in neoplastic cells [10], through expression and secretion of mutated PTEN, these mutations could eventually propagate their aberrant signal to the tumor microenvironment and interfere with the tumor-suppressive endogenous activity of wild-type PTEN. Although tissues can be differentially sensitive to loss of PTEN functions, epithelial cells are reportedly extremely susceptible to even partial reduction of PTEN levels [11, 12], leading to a more pronounced tumor-prone condition, and potentially a higher sensitivity to secreted mutated PTEN (Figure 1, bottom panel).

Regardless of the physiological processes that regulate expression and secretion of PTEN-Long, the second major discovery that Hopkins et al. have reported is their successful purification of active PTEN-Long, which is obviously of high relevance to the development of novel therapeutic approaches to cancer. Indeed, following the identification of the PTEN-long isoform, the authors went on to explore potential therapeutic applications by performing intraperitoneal (IP) injections of purified PTEN-Long into mice. Here they found that the protein was assimilated by several tissues and, by inhibiting Akt signaling, also induced an increase in blood glucose levels. Importantly, to further test the tumor-suppressive function of PTEN-Long on growing tumors, the authors performed in vivo treatments on a series of established xenograft models using multiple human cancer cell lines (U87MG, MDA-MB-468, HCC70, SUM149, HCT116). Notably, they found that IP injection of purified PTEN-Long led to uptake of the enzyme by tumor cells, which effectively caused tumor regression in most of the cell lines analyzed.

Taken together, these findings are exciting because they may offer a new and straightforward opportunity for cancer prevention and therapy: although research into the therapeutic effects of PTEN-Long is still in its infancy, we may dream of someday prescribing pills made of PTEN.

\section{Antonella Papa ${ }^{1}$, Ming Chen ${ }^{1}$, Pier Paolo Pandolfi ${ }^{1}$}

\begin{abstract}
${ }^{1}$ Cancer Genetics Program, Beth Israel Deaconess Cancer Center, Departments of Medicine and Pathology, Beth Israel Deaconess Medical Center, Harvard Medical School, Boston, MA 02115, USA

Correspondence: Pier Paolo Pandolfi

E-mail: ppandolf@bidmc.harvard.edu
\end{abstract}

\section{References}

1 Myers MP, Pass I, Batty IH, et al. Proc Natl Acad Sci USA 1998; 95:13513-13518.

2 Song MS, Carracedo A, Salmena L, et al. Cell 2011; 144:187-199.

3 Shen WH, Balajee AS, Wang J, et al. Cell 2007; 128:157-170.

4 Hollander MC, Blumenthal GM, Dennis PA. Nat Rev Cancer 2011; 11:289-301.

5 Song MS, Salmena L, Pandolfi PP. Nat Rev Mol Cell Biol 2012; 13:283-296.

6 Stambolic V, Suzuki A, de la Pompa JL, et al. Cell 1998; 95:29-39.

7 Hopkins BD, Fine B, Steinbach N, et al. Science 2013; 341:399-402.

8 Putz U, Howitt J, Doan A, et al. Sci Signal 2012; 5:ra70.

9 Azmi AS, Bao B, Sarkar FH. Cancer Metastasis Rev 2013 May 25. doi:10.1007/ s10555-013-9441-9

10 Bonneau D, Longy M. Hum Mutat 2000; 16:109-122.

11 Alimonti A, Carracedo A, Clohessy JG, et al. Nat Genet 2010; 42:454-458.

12 Berger AH, Knudson AG, Pandolfi PP. Nature 2011; 476:163-169. 